\title{
MAGIS-100: 100 m Atomic Interferometry at Fermilab
}

\section{Rob Plunkett, Fermilab - for the MAGIS-100 Collaboration}

\section{Chicago Quatum Exchange 10/27/2019 FERMILAB-POSTER-19-131-QIS}

\section{Atom Interferometry}

- Laser pulses act as beam splitters and mirrors for atomic wavefunction

- Highly sensitive to accelerations (or to time-

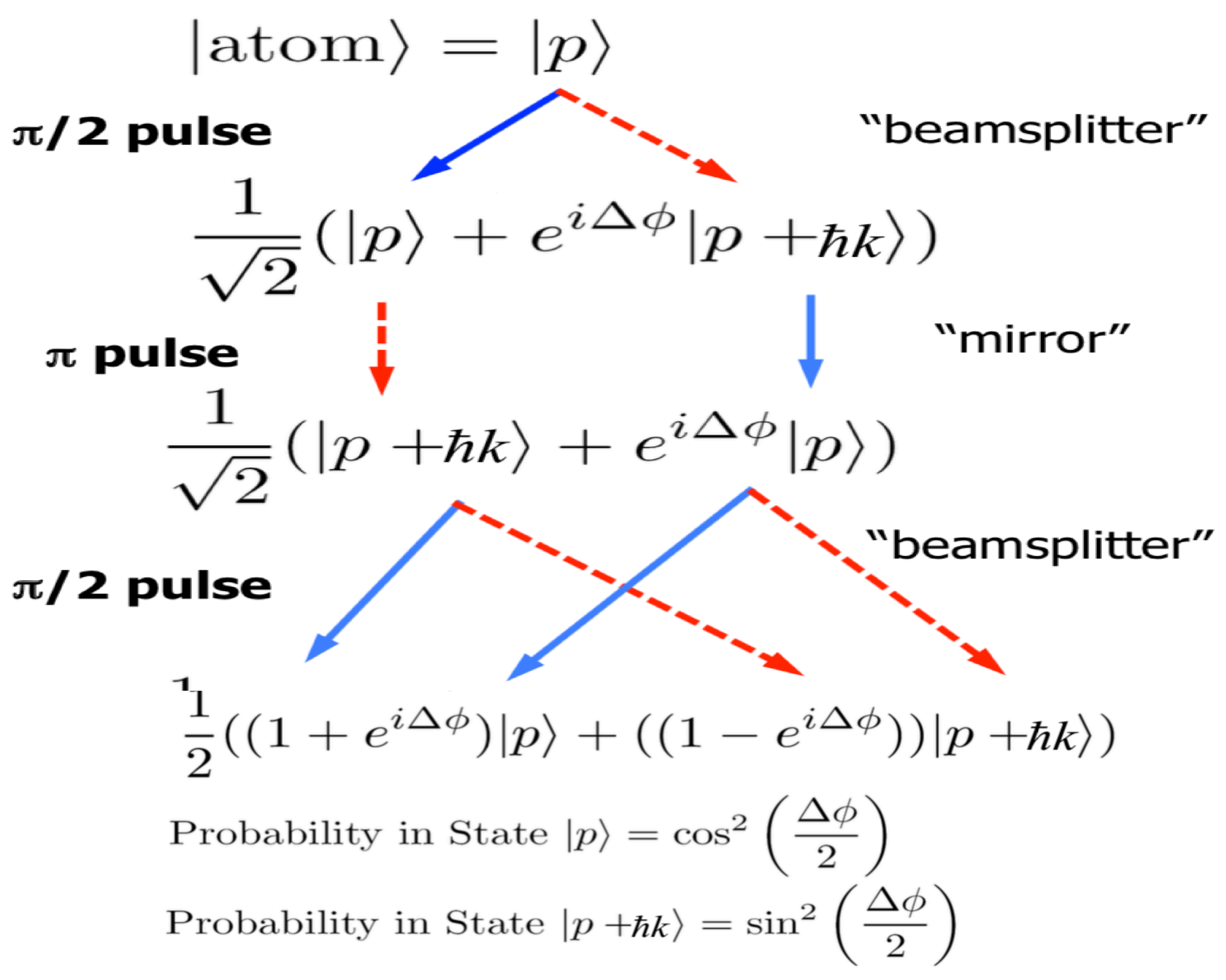

\section{Extreme Quantum Science}

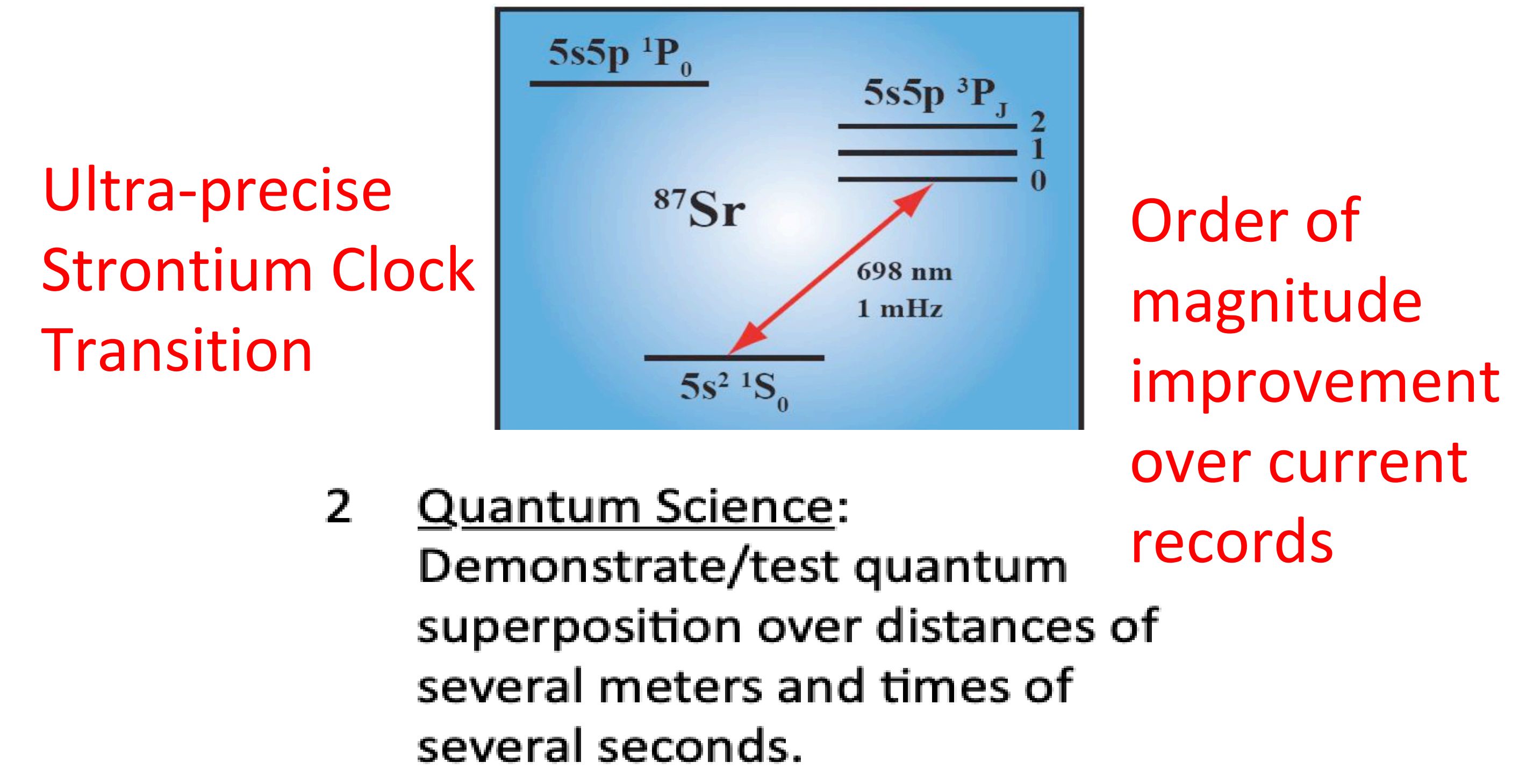

Prototype Gravitational Wave Detector

Inspiral produces $\sim 1 \mathrm{~Hz} \mathrm{GW}$ for days to weeks.

Below LIGO cutoff - ideal for

Atom Interferometry

Next generation of 1-4 km

detector will be sensitive to

known sources

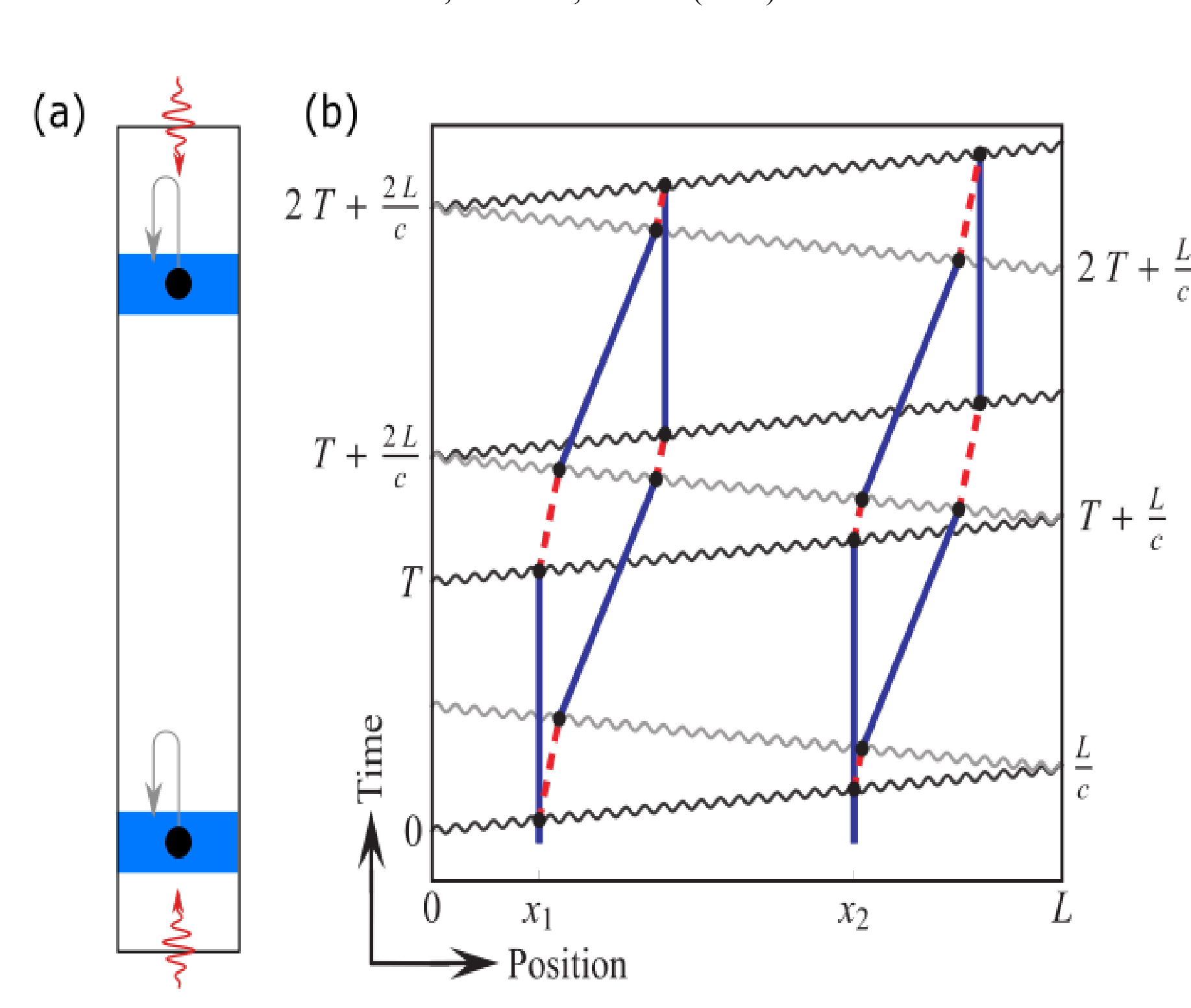

Gradiometer with 2 interferometers

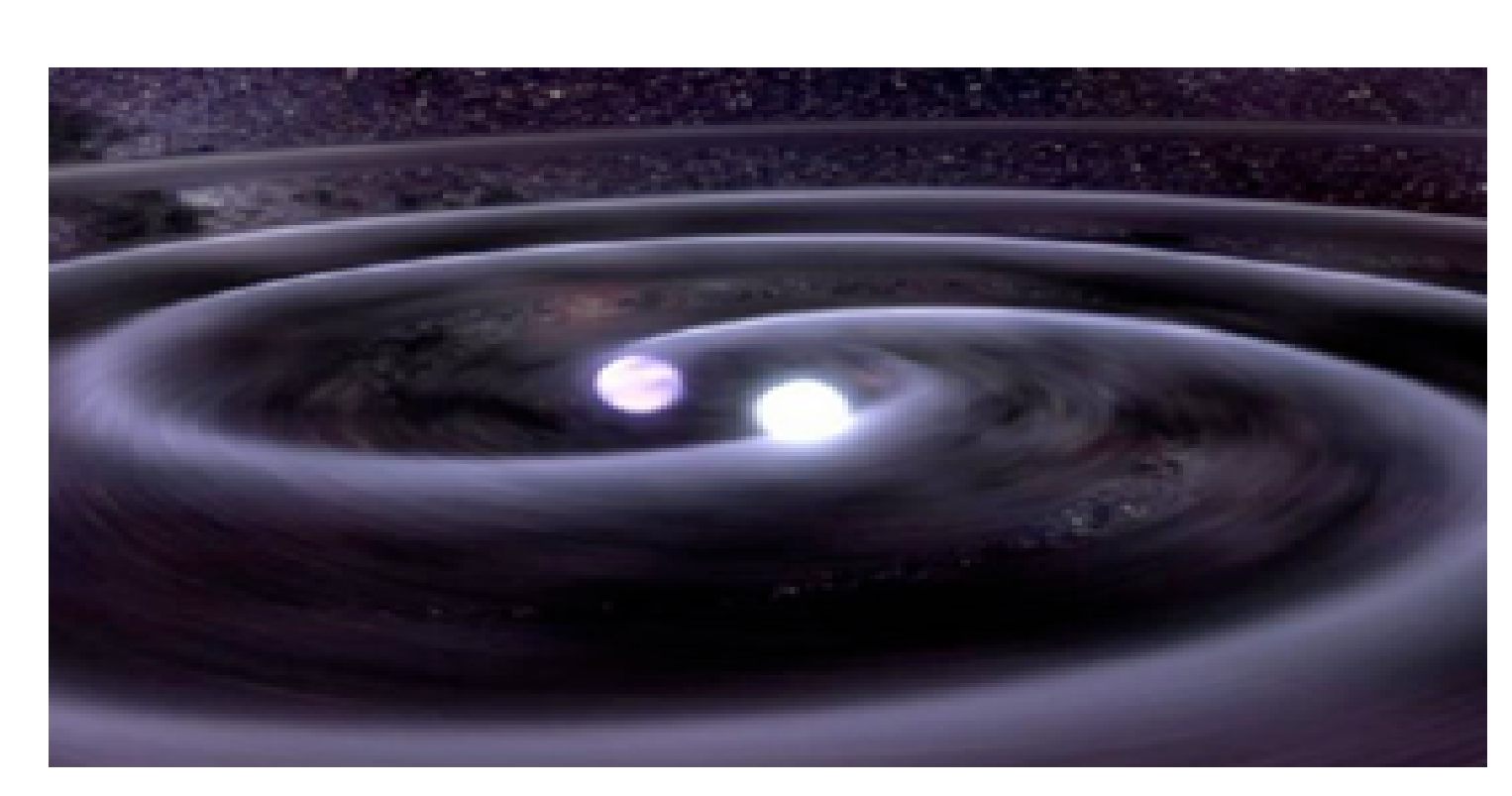

GW changes light travel time
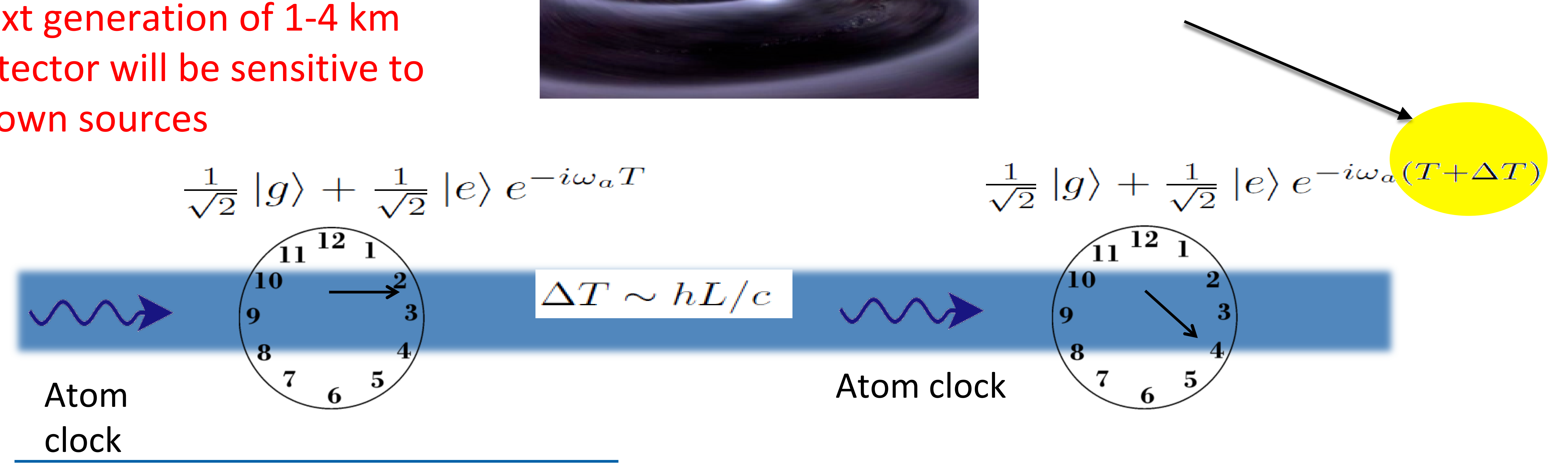

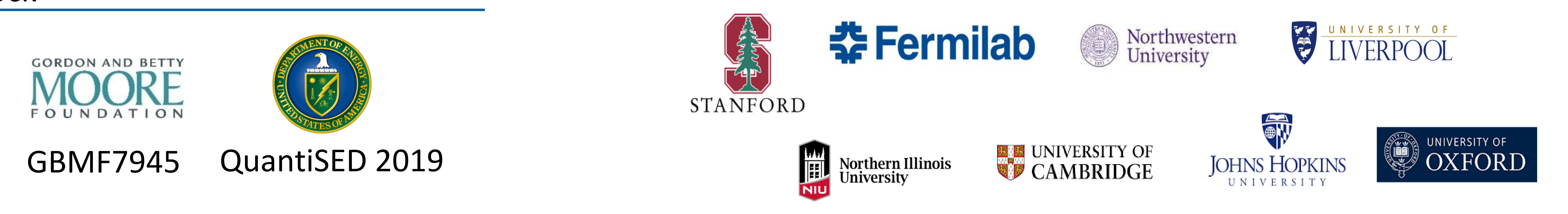

\section{Ultra-light Dark Matter}

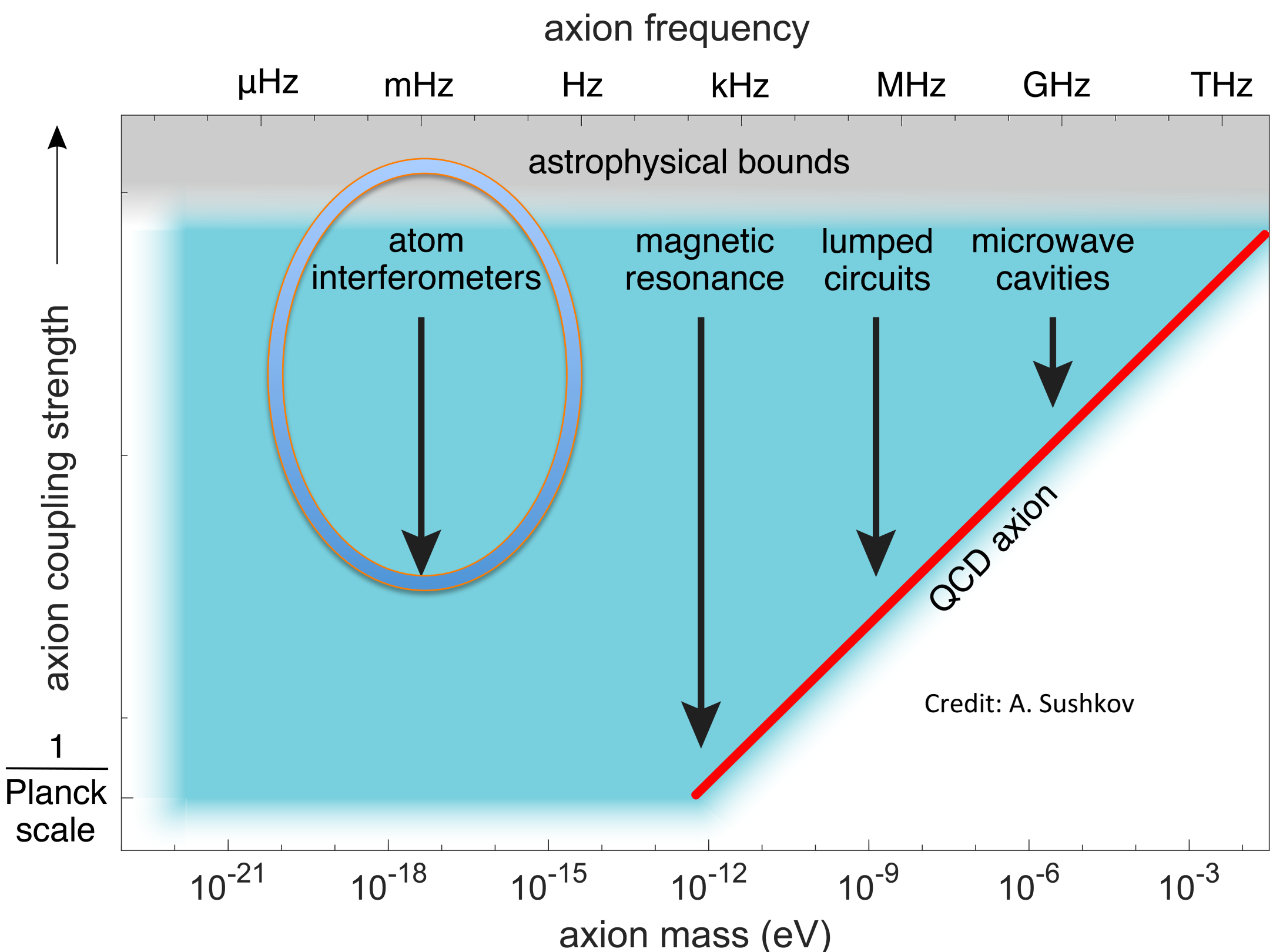

gire from DOE Dark Matter Research Needs Report, 2018

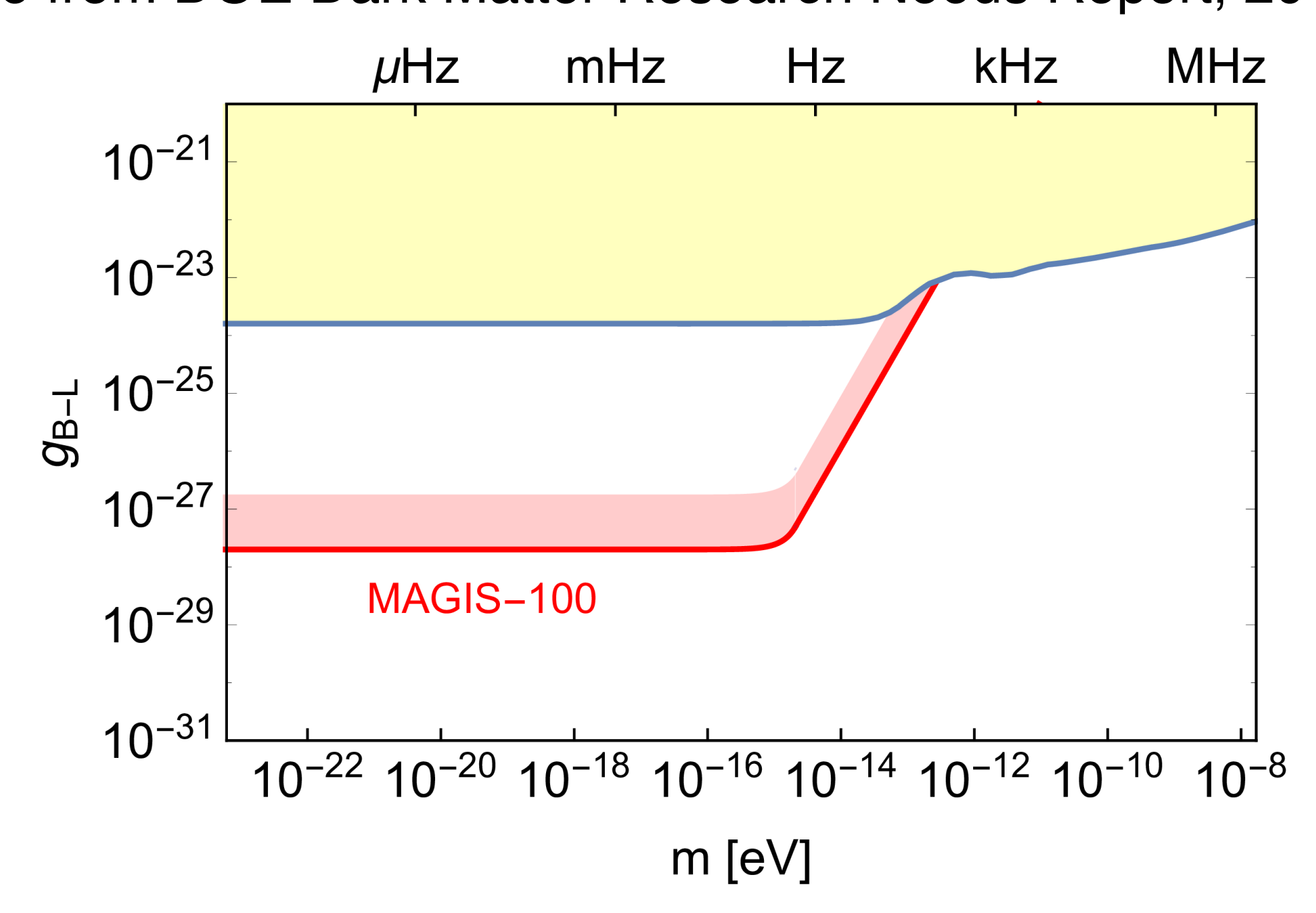

Expected MAGIS-100 B-L dark matter sensitivity

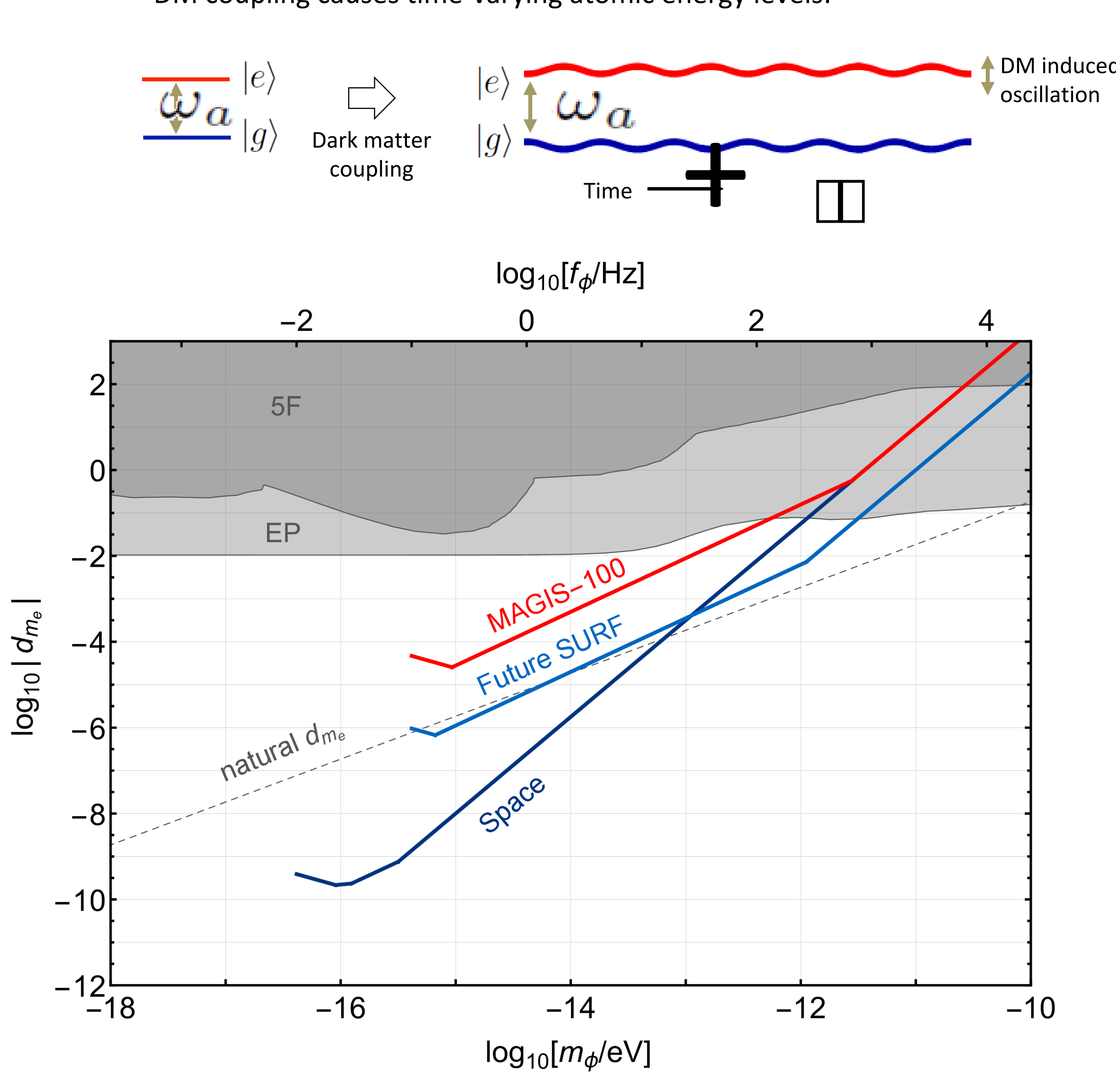

MAGIS-100: Bringing Large Scale Interferometry to

\section{Fermilab}

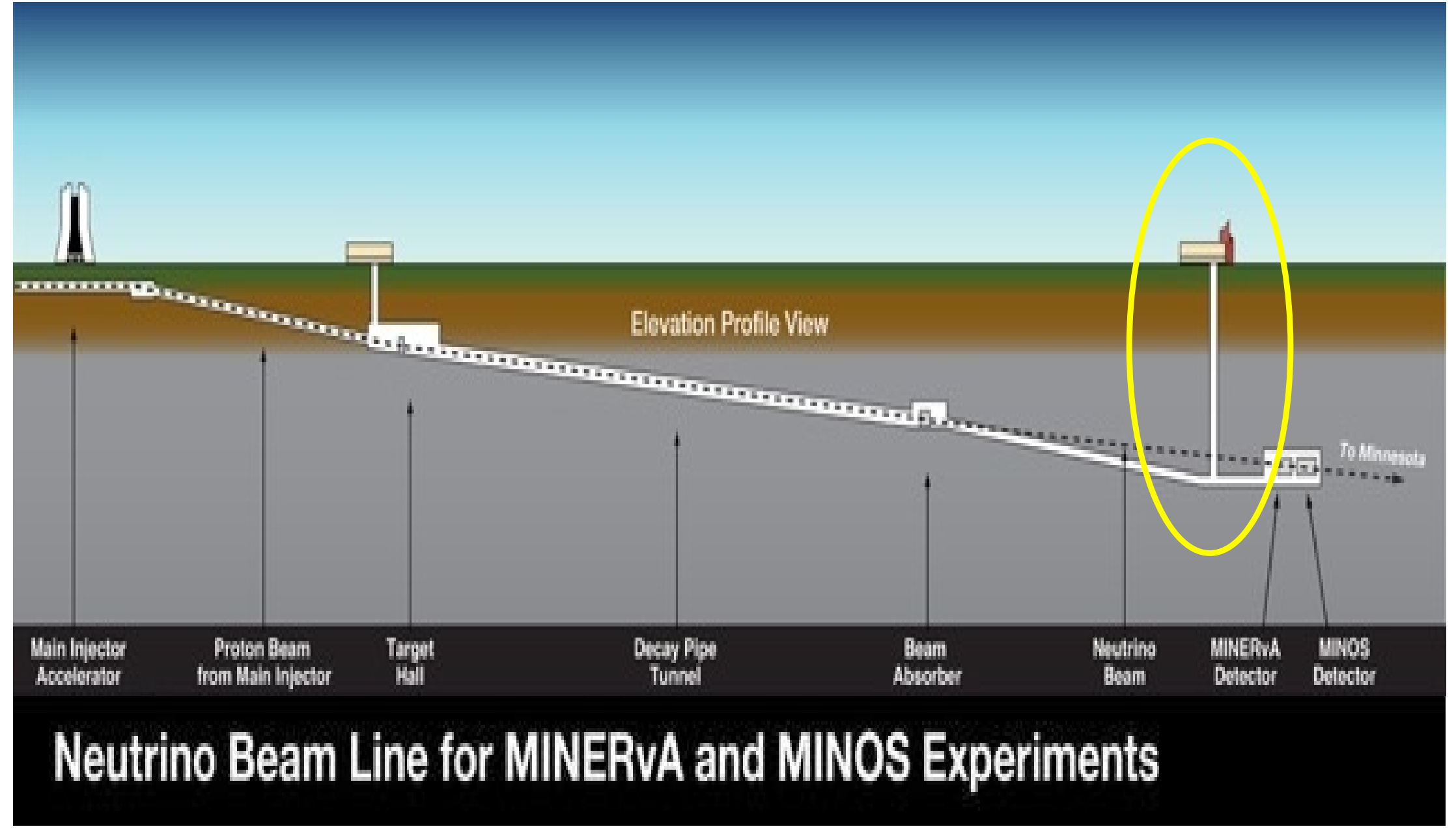

Use existing $100 \mathrm{~m}$ shaft from NuMI/MINOS program Equipped surface building because underground experiments still active.

Serves both to study fundamental physics and as prototype for longer baseline ( $\mathrm{km}$ scale ) in future. Installation goal late 2021.

\section{Details of Atom Source (one of 3)}

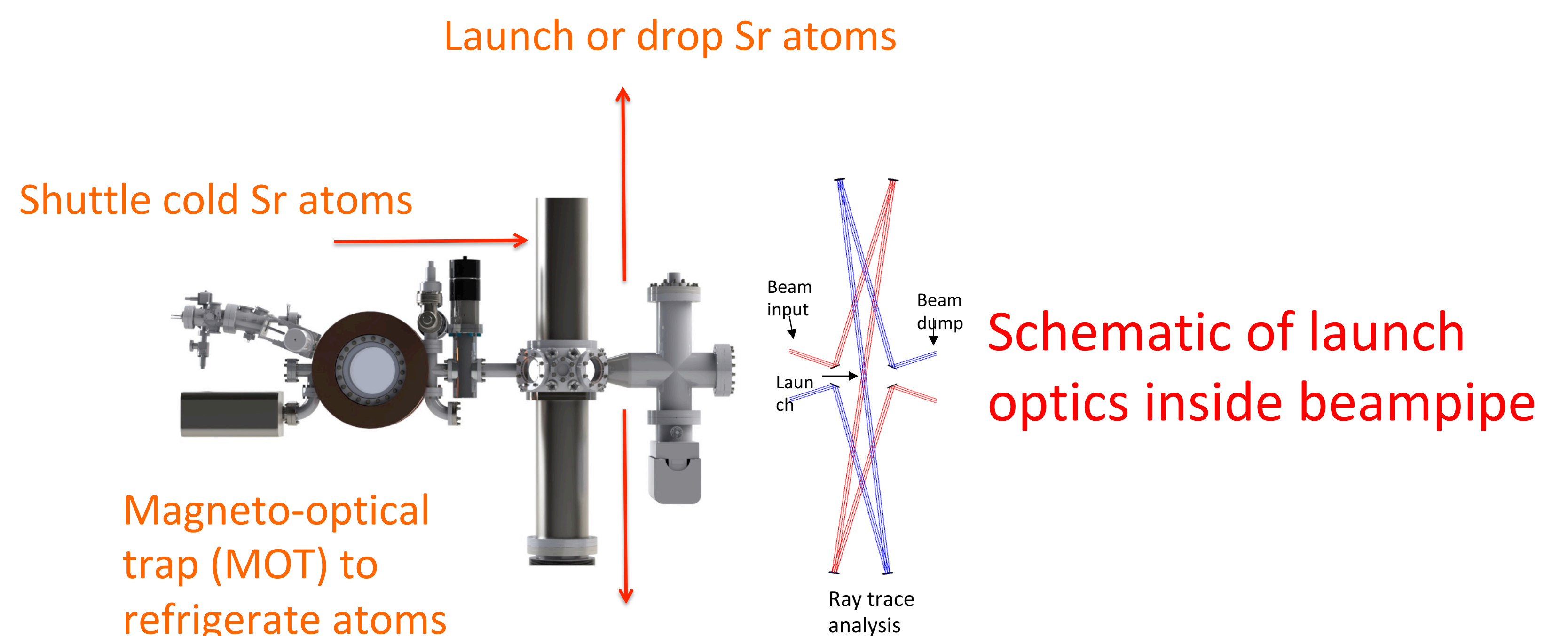

This manuscript has been authorized by Fermi Research Alliance, LLC under Contract No. DE-AC02-07CH11359 with the United States Department of Energy, Office of Science, Office of High Energy Physics. This work is supported in part by the U.S. Department of Energy, Office of Science, QuantiSED intitiative. Major support has come from the Gordon and Betty Moore Foundation under grant GBMF7945. 